\title{
Properly assessing CD133 as a risk factor for poor prognosis in patients with hepatocellular carcinoma after resection
}

\author{
Hao-Feng $\mathrm{Lu}^{1,2} \cdot$ Wei-Ping Yuan ${ }^{2} \cdot \mathrm{Mi} \mathrm{Li}^{1}$ •

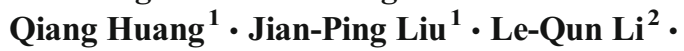 \\ Jian-Hong Zhong ${ }^{2}$
}

Received: 11 May 2015 / Accepted: 26 May 2015 / Published online: 31 May 2015

(C) International Society of Oncology and BioMarkers (ISOBM) 2015

Our understanding of the pathophysiology of hepatocellular carcinoma (HCC) onset and progression is as poor as the prognosis of many patients with the disease, even when they can undergo curative hepatic resection. For patients with large and/or multinodular HCC after hepatic resection, 5-year overall survival (OS) is lower than $50 \%$ and disease-free survival (DFS) is lower than $30 \%[1,2]$. This highlights the need to identify reliable risk factors for poor OS and DFS in patients following resection.

In a recent issue of Tumour Biology, Zhong and coworkers [3] meta-analyzed 21 retrospective studies involving $2592 \mathrm{pa}-$ tients with $\mathrm{HCC}$ in an effort to determine whether overexpression of the cancer stem cell (CSC) marker CD133 is associated with poor prognosis after resection. Increasing evidence suggests that CSCs are the cancer-initiating cells responsible for carcinogenesis and that they also contribute to cancer resistance and metastasis [4, 5]. Zhong et al. found that CD133 overexpression was significantly associated with low tumor differentiation, advanced tumor stage, presence of vascular invasion and thrombosis, and poor OS and DFS [3]. These results are similar to a previous meta-analysis by other authors of 12 retrospective studies involving 1344 patients [6].

Hao-Feng Lu, Wei-Ping Yuan and Mi Li contributed equally to this work.

Jian-Hong Zhong

zhongjianhong66@163.com

1 The Second Hepatobiliary Surgery Department, Affiliated Hospital of Yangtze University, Jingzhou 434000, People's Republic of China

2 Hepatobiliary Surgery Department, Affiliated Tumor Hospital of Guangxi Medical University, He Di Rd. \#71, Nanning 530021, People's Republic of China
While the meta-analysis by Zhong et al. [3] provides important new information about the association of CD133 overexpression with HCC clinicopathology and outcomes, its conclusions should be treated with caution in light of several limitations. First, the authors systematically searched only the databases of PubMed, Chinese National Knowledge Infrastructure, and Wanfang. Since HCC is particularly prevalent throughout the Asia-Pacific region, it is possible that including Japanese, Taiwanese, and Korean databases would turn up additional eligible studies. Not surprisingly, then, more than $90 \%$ of the patients in the study by Zhong et al. [3] were Chinese. This raises the possibility that the meta-analysis results are not valid for other ethnic groups [7].

Although the meta-analysis nominally involved 21 studies, the number used to meta-analyze each outcome was much smaller. According to the authors themselves, only ten of the included studies $(48 \%)$ reported data on the association between CD133 overexpression and OS, while only five (24\%) reported data on the association between CD133 overexpression and DFS. Of these five studies, one reported data only on recurrence rate [8] and another only on DFS [9].

The authors address the issue of publication bias in the article, but the issues they mention seem irrelevant. In addition, they assess risk of publication bias using Egger's and Begg-Mazumdar approaches, but Harbord's modified test or Peters' method [10] are likely much more appropriate since they can detect an excess of statistically significant results in meta-analyses.

The authors also fail to document their systematic literature search in adequate detail. It is surprising that they failed to identify any eligible studies when searching in "other sources" (mentioned in the flowchart), though they never define what these sources were. They did not indicate the reasons why 
they excluded articles from the meta-analysis, which further increases the risk of bias.

While Zhong et al. [3] address important questions in their meta-analysis of CD133 as a risk factor of poor prognosis in patients with HCC, the shortcomings in their methods and reporting should serve as a lesson for future studies in order to ensure that researchers and clinicians have access to the most reliable and comprehensive evidence base for their work.

\section{References}

1. Zhong JH, Rodriguez AC, Ke Y, Wang YY, Wang L, Li LQ. Hepatic resection as a safe and effective treatment for hepatocellular carcinoma involving a single large tumor, multiple tumors, or macrovascular invasion. Medicine (Baltimore). 2015;94(3):e396.

2. Zhong JH, Lu SD, Wang YY, Ma L, Li LQ. Intermediate-stage HCC-upfront resection can be feasible. Nat Rev Clin Oncol. 2015;12(5):295.
3. Zhong $\mathrm{C}, \mathrm{Wu}$ JD, Fang MM, Pu LY. Clinicopathological significance and prognostic value of the expression of the cancer stem cell marker CD133 in hepatocellular carcinoma: a meta-analysis. Tumour Biol. 2015. doi:10.1007/s13277-015-3487-y.

4. Jordan CT, Guzman ML, Noble M. Cancer stem cells. N Engl J Med. 2006;355(12):1253-61.

5. Bjerkvig R, Tysnes BB, Aboody KS, Najbauer J, Terzis AJ. Opinion: the origin of the cancer stem cell: current controversies and new insights. Nat Rev Cancer. 2005;5(11):899-904.

6. Ma YC, Yang JY, Yan LN. Relevant markers of cancer stem cells indicate a poor prognosis in hepatocellular carcinoma patients: a meta-analysis. Eur J Gastroenterol Hepatol. 2013;25(9):1007-16.

7. Pan Z, Trikalinos TA, Kavvoura FK, Lau J, Ioannidis JP. Local literature bias in genetic epidemiology: an empirical evaluation of the Chinese literature. PLoS Med. 2005;2(12):e334.

8. Song W, Li H, Tao K, et al. Expression and clinical significance of the stem cell marker CD133 in hepatocellular carcinoma. Int J Clin Pract. 2008;62(8):1212-8.

9. Chan AW, Tong JH, Chan SL, Lai PB, To KF. Expression of stemness markers (CD133 and EpCAM) in prognostication of hepatocellular carcinoma. Histopathology. 2014;64(7):935-50.

10. Ioannidis JP, Trikalinos TA. An exploratory test for an excess of significant findings. Clin Trials. 2007;4(3):245-53. 